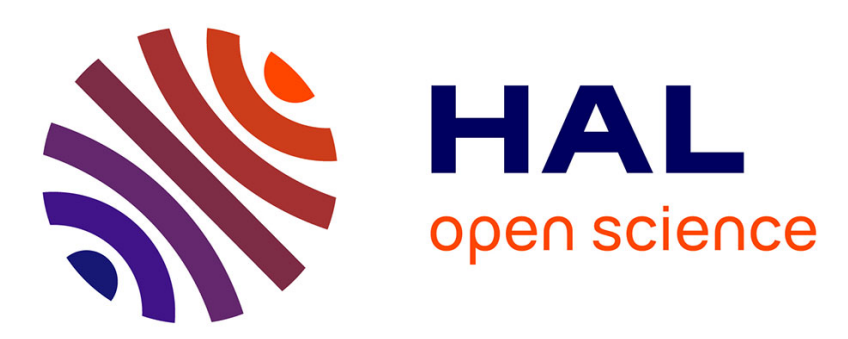

\title{
A Two-stage Stochastic Programming Approach for Aircraft Landing Problem
}

Ming Liu, Bian Liang, Feifeng Zheng, Chengbin Chu, Feng Chu

\section{To cite this version:}

Ming Liu, Bian Liang, Feifeng Zheng, Chengbin Chu, Feng Chu. A Two-stage Stochastic Programming Approach for Aircraft Landing Problem. 15th International Conference on Service Systems and Service Management (ICSSSM 2018), Jul 2018, Hangzhou, China. 10.1109/ICSSSM.2018.8465107 . hal02077980

\section{HAL Id: hal-02077980 \\ https://hal.science/hal-02077980}

Submitted on 25 Mar 2019

HAL is a multi-disciplinary open access archive for the deposit and dissemination of scientific research documents, whether they are published or not. The documents may come from teaching and research institutions in France or abroad, or from public or private research centers.
L'archive ouverte pluridisciplinaire HAL, est destinée au dépôt et à la diffusion de documents scientifiques de niveau recherche, publiés ou non, émanant des établissements d'enseignement et de recherche français ou étrangers, des laboratoires publics ou privés. 


\title{
A Two-stage Stochastic Programming Approach for Aircraft Landing Problem
}

\author{
$1^{\text {st }}$ Ming Liu \\ $2^{\text {nd }}$ Bian Liang \\ $3^{\text {rd }}$ Feifeng Zheng \\ School of Economics \& Management School of Economics \& Management Glorious Sun School of Business \&Management \\ Tongji University \\ Tongji University \\ Shanghai, China \\ Shanghai, China \\ mingliu@tongji.edu.cn \\ liangbian@tongji.edu.cn \\ Donghua University \\ Shanghai, China \\ ffzheng@dhu.edu.cn
}

\author{
$4^{\text {th }}$ Chengbin Chu \\ Laboratoire Génie Industriel, Centrale Supélec \\ Université Paris-Saclay \\ Châtenay-Malabry, France \\ chengbin.chu@ecp.fr
}

\author{
$5^{\text {th }}$ Feng Chu \\ Laboratory IBISC \\ University of Evry-Val d'Essonne \\ Evry, France \\ feng.chu@ibisc.univ-evry.fr
}

\begin{abstract}
This paper considers a two-stage stochastic programming problem for airport runway scheduling under the uncertainty of arrival time on a single runway. The goal of airport runway scheduling is to schedule a set of aircrafts in a given time horizon and minimize a corresponding objective while satisfying separation requirements as well as other practical constraints. In order to boost runway elasticity and throughout, a mess of unpredictable factors, such as weather, pilot behavior and airport surface traffic, should be take into consideration by airport regulator. The arrival scheduling problem at airport can be decomposed into sequential decision problem, where the first stage determines the sequence of aircraft weight class, while the individual flight is assigned to positions in the weight class sequence in the second stage. The main mission of this work is to identify an optimal schedule involving the arrival time of flight is stochastic under different scenarios. A stochastic aircraft landing problem (SALP) formulation based on time-dependent traveling salesman problem (TDTSP) is proposed. Then a sample average approximation (SAA) algorithm is developed to solve this stochastic programming and the efficacy is verified by experimental result.
\end{abstract}

Index Terms-Airport runway scheduling, Stochastic programming, Sample average approximation.

\section{INTRODUCTION}

Runway system is the most critical component of airport, especially in major international airports, which is known admitted as the prime reason of delay in the departure/arrival process and contributes to the congestion in the airport terminal area as a result of restricted runway capacity and inefficient operations. The total air transportation demand is growing steadily at a rate between three and five percent in spite of the recent economic recession [1]. Beyond question, the increasing traffic will causes high congestion in the terminal areas due to the limited capacity of runway system that defined as the number of runway operations (i.e., aircraft take-off and landing) in a unit time. Airport capacity can be increased by building a new runway, however, it's a expensive investment to the infrastructure. In contrary, improving the existed aircraft take-off and landing schedules is a cost-efficient method to enlarge the capacity of the runway system rather than builds a new one. The runway scheduling problem (RSP) can be defined as follows. The service time on a runway for each plane, in a given set of flights, whether arrivals, departures or a mixed-mode, should be decided in a specified planning horizon. Meanwhile, a predetermined time window and the separation requirement between the front and back aircrafts should be satisfied. In this work, the efficient scheduling of landing aircraft on a single runway is mainly discussed. The aircraft landing problem (ALP) is a subproblem of the more general aircraft scheduling problem (e.g., mixed-mode and parallel runway scheduling problem considering takeoff and departure simultaneously or respectively). The goal of ALP is to minimize the total separation times (i.e., the throughout of a runway) and the delay times for all the landing aircrafts while maintaining the separation requirements, since the arrival time for each flight realization. Table II shows a matrix of aircraft weight class-dependent minimum separation times, which comes from Ghoniem et al. [2].

The first mixed-integer programming (MIP) model for the ALP on a single runway was presented by Abela et al. [3], and a branch-and-bound (B\&B) algorithm was developed to solve the single-runway ALP. Beasley et al. [4] generalized the single-runway to multiple runway and the extension is the most cited MIP model of the ALP to date. The ALP formulation is further extended by Pinol and Beasley [5], which considers the runway-dependent time window and separation times. Briskorn and Stolletz [6] proposed a modification of the formulation based on [4] that explicitly takes the aircraft class into consideration, and developed four polynomial heuristic algorithms to solve it. A variety of ALPs have been resolved by means of dynamic programming (DP) approach in the literature, and many of these studies assume that the aircraft within a weight class can be sequenced, typically by establishing that there exist an optimal solution to the ALP that 
always satisfies this property. Dear and Sherif [7] as well as Balakrishnan and Chandran [8] present a DP model for the ALP with a constrained-position-shifting (see Psaraftis [9] for more details) assumption and making use of the classdependent separation times to reduce the problem complexity. Recently, Lieder et al. [10] develop a DP approach combined with a state-space reduction using a dominance criterion, which is able to create optimal landing schedules on multiple independent runways. Bennell et al. [1] investigates DP and local search implementation for both static and dynamic ALP. Also, a lot of authors empoy heuristic method as a solution approach (e.g., Bianco et al. [11], Beasley et al. [12] and Faye [13]). Moreover, $\mathrm{Ng}$ et al. [15] recently consider the robust aircraft sequencing and scheduling problem under the uncertainty of arrival and departure delays, and a artificial bee colony algorithm is designed to solve it based on min-max regret approach. The literature for ALP is summarized in Table I.

TABLE I: Summary of representative literature of the aircraft landing problem.

\begin{tabular}{lllll}
\hline Literature & Runway & $\begin{array}{l}\text { TDTSP } \\
\text { model }\end{array}$ & Arrival time & Method \\
\hline$[3]$ & single & no & deterministic & branch-and-bound \\
{$[4]$} & multiple & no & deterministic & heuristic algorithm \\
{$[6]$} & single & no & deterministic & heuristic algorithm \\
{$[8]$} & single & no & deterministic & dynamic programming \\
{$[10]$} & multiple & no & deterministic & dynamic programming \\
{$[15]$} & multiple & no & deterministic & artificial bee colony al- \\
& & & & gorithm \\
This work & single & yes & stochastic & SAA algorithm \\
\hline
\end{tabular}

To our best knowledge, the deterministic, dynamic and robust ALP have been extensively studied in runway operations. However, ALP with the consideration of uncertainty is rarely studied. This work, motivated by above research gap, proposes a two-stage stochastic mathematical formulation of ALP (SALP) to incorporate the uncertain factors (i.e., arrival times of aircraft) into the ALP. This can in favour of improving the runway availability and reducing flight delays based on the feature that practical aircraft arrival time is uncertain.

The contributions of this research can be summarised as follows: First, a two-stage stochastic ALP formulation is proposed, which incorporates the uncertainty of arrival time into the scheduling process. In this work, minimising the total separation times and the delay times of aircraft is considered in the SALP model. Second, coupled with the number of aircraft weight class and scenarios for each actual arrival time, this poses a very large-scale problem compared with deterministic one. this article presents a sample average approximation algorithm, which can be used to solve this problem and provide information on the quality of the solution. Third, Numerical experiments are conducted to evaluate the effectiveness of the proposed mathematical model and resolution approach.

The remainder of this article is organized as follows. in the next section, a mathematical formulation of the SALP is given. The solution procedure based on the SAA is introduced in section III, In section IV, we dedicated to conduct the numerical experiments to demonstrate the efficacy for the proposed solution method. Finally, section V concludes this work and associated directions for future research are summarized.

TABLE II: Separation Requirement (in seconds).

\begin{tabular}{ccccc}
\hline & & \multicolumn{3}{c}{ Trailing aircraft } \\
\cline { 3 - 5 } & & Heavy & Large & Small \\
\hline \multirow{4}{*}{ Lading aircraft } & Heavy & 99 & 133 & 196 \\
& Large & 74 & 107 & 131 \\
& Small & 74 & 80 & 98 \\
\hline
\end{tabular}

\section{Problem Formulation}

In this section, we present a two-stage stochastic mixedinteger $0-1$ formulation of the single runway aircraft landing problem based on time-dependent TSP proposed by Picard and Queyranne [16] (see Bigras et al. [17] for more details).

\section{A. Assumption}

Before the mathematical model of stochastic aircraft landing problem (SALP) is established, several assumptions should be made.

1) The SALP schedules are solely focused on the aircraft landing operation decisions without involving the ground operations, terminal air traffic control and take-off activities.

2) The length of the single runway is long enough to accommodated different aircraft weight class, which requires different minimal length of the runway to landing operation.

3) Every aircraft can't landing before a realization arrival time, and the latest landing time for each is greater than the given planning horizon (i.e., 30-40 minutes).

\section{B. The SALP model}

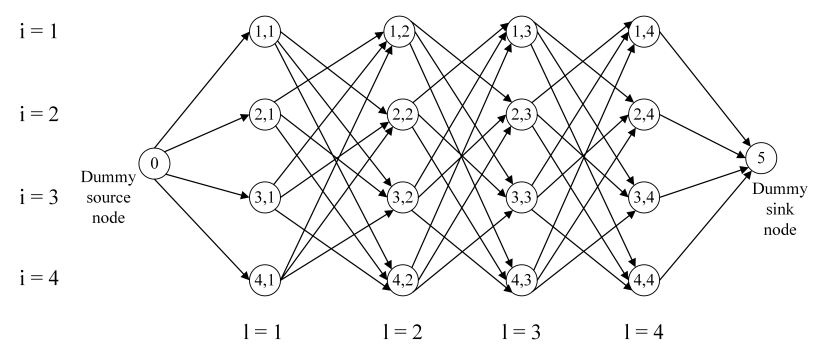

Fig. 1: The multipartite network for a 4 flights landing problem.

The time-dependent traveling salesman problem (TDTSP) is a version of the classical traveling salesman problem (TSP), where the arc costs between node $i$ and node $j$ depends on which time period node $i$ is visited [17]. TDTSP formulations have been widely applied to a single machine scheduling problem (SMSP) with sequence dependent set-up 
time. The objective of first-stage problem in SALP aims to find a sequence of aircraft weight class that minimizes the total separation requirements of completing all the landing operations. This character can be expressed as a traveling salesman problem (TSP) as follows. Consider a direct graph where each alternate aircraft is regarded as a node with arcs connecting each pair of nodes (i.e., landing aircraft), and let the arc costs define separation times between sequential flights. It is easy to indicate that the optimal TSP tour on this time-dependent network would correspond to a sequence of aircraft weight class that minimizes the total separation requirements. The second-stage problem considers assigning individual aircraft to specific locations in the sequence, since the actual arrival times realization. The assignment process in second-stage problem requires explicit representation of the location information received from the solutions of first-stage problem. On account of the need request for the location information in the second-stage, the first-stage problem can be represented as a location-dependent TSP (LDTSP), which is also known as time-dependent TSP. The cost of flowing an arc rests on the aircrafts (i.e., nodes) it connects and the location (i.e., time) in the sequence. Figure 1 represents a multipartite network for a 4 flights landing problem, and the goal of the LDTSP can be seen as a shortest path problem on a multipartite network with complicating constraints that are used to force the path to visit every node once and only once.

This problem can be addressed more formally as follows. Let $G(\mathcal{N}, \mathcal{A})$ be an oriented graph with the node set $\mathcal{N}=$ $\left\{n_{i, l}: i=1,2, \ldots, n ; l=1,2, \ldots, n\right\} \cup n_{0}, n_{n+1}$, where a node consists of a particular flight $i$ and location $l$, and the $n_{0}$ and $n_{n+1}$ represents dummy source and sink nodes, respectively. The set of arcs is defined as $\mathcal{A}=\left\{\left(n_{i, l}, n_{j, l+1}\right): i=\right.$ $1,2, \ldots, n ; j=1,2, \ldots, n ; l=1,2, \ldots, n\} \cup\left\{\left(n_{0}, n_{i, 1}\right): i=\right.$ $1,2, \ldots, n\} \cup\left\{\left(n_{i, n}, n_{n+1}\right): i=1,2, \ldots, n\right\}$.

Input Parameters:

$K$ : set of the weight class of the aircraft, indexed by $k$, and $|K|$ denotes the number of aircraft classes.

$N$ : set of landing flights that can be scheduled in a given planning time, indexed by $i, j$, and $n$ denotes the number of flight will be scheduled in a given planning horizon.

$L$ : set of location in the flight landing sequence, indexed by $l$, where $l \in L$, and $|L|$ denotes the number of locations, $|L|=n$.

$S$ : set of scenarios corresponding to demand realizations, indexed by $s$.

$N_{k}$ : the set of aircraft belong to aircraft weight class $k$, where $k \in K$.

$a_{k}$ : the number of landing flight belong to aircraft class $k$, where $k \in K$.

$T_{i}^{s}$ : the realization landing time of flight $i$ under scenario $s$, where $i \in N, s \in S$.

$\mathbf{T}^{s}$ : the vector of realization landing time for all flights under scenario $s$, where $s \in S$.

$p^{s}$ : probability for realization of scenario $s$, where $s \in S$.

$d_{i, j}$ : the separation requirements between weight class of front and back aircrafts, where $i, j \in N$. As in Table II, $d_{i, j}$ is determined by the aircraft weight class of of the leading and following aircraft $i$ and $j$.

\section{Decision Variables:}

$x_{l, k}$ : (first-stage variable) $=1$ if aircraft class $k$ is assigned to location $l,=0$ otherwise, where $l \in L, k \in K$.

$c_{l}$ : (first-stage variable) denotes the time of location $l$, where $l \in L$.

$y_{i, l}^{s}:$ (second-stage variable) $=1$ if flight $i$ is assigned to location $l,=0$ otherwise, where $i \in N, l \in L$.

$z_{0, j}^{0, s}:$ (second-stage variable) $=1$, if there is flow on arc, which outflows from dummy source node $n_{0}$. where $j \in N$, $s \in S$.

$z_{i, j}^{l, s}$ : (second-stage variable) $=1$ if there is flow on arc $\left(n_{i, l}, n_{j, l+1}\right)$ in scenario $s$, where $i \in N, j \in N, s \in S$.

$v_{n+1}^{s}:($ second-stage variable) $=1$, if there is flow on arc, which inflows to dummy sink node $n_{n+1}$. where $i \in N, s \in S$.

$t_{i}^{s}$ : (second-stage variable) denotes the landing time of flight $i$ in scenario $s$, where $i \in N, s \in S$.

For conciseness, we use bold notations to denote the corresponding vectors of parameters or variables in the remainder. Given the above notation, a compact representation of the corresponding deterministic equivalent formulation of the twostage stochastic integer problem (MIP) based on a LDTSP model can be described as follows. The objective function of the first-stage problem reads as

$$
\min \mathbb{E}(\mathbf{x}, \omega)=c_{n}+\sum_{s \in S} p^{s} \cdot Q^{s}\left(\mathbf{x}, \mathbf{T}^{s}\right)
$$

subject to

$$
\begin{gathered}
\sum_{k \in K} x_{k, l}=1, \quad l \in L \\
\sum_{l \in L} x_{k, l}=a_{k}, \quad k \in K \\
\mathbf{x} \in\{0,1\}^{|K| \times|L|}
\end{gathered}
$$

In the second-stage problem, for each scenario $s \in S$, the objective (also known as recourse function) is written as

$$
Q^{s}\left(\mathbf{x}, \mathbf{T}^{s}\right)=\min \sum_{l \in L} t_{l}^{s}-\sum_{i \in N} T_{i}^{s}
$$

subject to

$$
\begin{gathered}
\sum_{i \in N_{k}} \sum_{j \in N} z_{i, j}^{l, s}=x_{k, l}, \quad l \in L, l \neq n, k \in K, s \in S \\
\sum_{i \in N_{k}} z_{i, n+1}^{n, s}=x_{k, n}, \quad k \in K, s \in S \\
\sum_{j \in N} z_{0, j}^{0, s}=1, \quad s \in S \\
\sum_{i \in N} z_{j, i}^{1, s}=z_{0, j}^{0, s}, \quad j \in N, s \in S
\end{gathered}
$$




$$
\begin{aligned}
& \sum_{i \in N} z_{i, j}^{l, s}=\sum_{i \in N} z_{j, i}^{l+1, s}, \quad l=1, \ldots, n-2, j \in N, s \in S \\
& \sum_{i \in N} z_{i, j}^{n-1, s}=z_{j, n+1}^{n, s}, \quad j \in N, s \in S \\
& z_{0, j}^{0, s}+\sum_{i \in N} \sum_{l \in L \backslash\{n\}} z_{i, j}^{l, s}=1, \quad j \in N, s \in S \\
& \sum_{j \in N} z_{i, j}^{l, s}=y_{i, l}^{s}, \quad i \in N, l \in L, l \neq n, s \in S \\
& z_{i, n+1}^{n, s}=y_{i, n}^{s}, \quad i \in N, s \in S \\
& \sum_{i \in N} z_{i, j}^{l, s}=y_{j, l+1}^{s}, \quad j \in N, l \in L, l \neq n, s \in S \\
& z_{0, j}^{0, s}=y_{j, 1}^{s}, \quad j \in N, s \in S \\
& d_{0, j} \cdot z_{0, j}^{0, s} \leq c_{1}, \quad i \in N, s \in S \\
& c_{l-1}+\sum_{i \in N} \sum_{j \in N} d_{i, j} \cdot z_{i, j}^{l-1, s} \leq c_{l}, \quad l \in L, l \neq 1, s \in S \\
& d_{0, j} \cdot z_{0, j}^{0, s} \leq t_{1}, \quad i \in N, s \in S \\
& t_{l-1}^{s}+\sum_{i \in N} \sum_{j \in N} d_{i, j} \cdot z_{i, j}^{l-1, s} \leq t_{l}^{s}, \quad l \in L, l \neq 1, s \in S \\
& \sum_{i \in N} T_{i}^{s} \cdot y_{i, l}^{s} \leq t_{l}^{s}, \quad l \in L, s \in S \\
& \mathbf{c}, \mathbf{t}^{s}, \mathbf{y}^{s} \geq 0, \mathbf{z}^{s} \in\{0,1\}^{|N| \times|L|}, \quad s \in S
\end{aligned}
$$

In this model, the objective function (1) minimizes the total separation requirement and delay time for each aircraft. The first-stage constraints (2), (3) and 0-1 requirements (4) ensure that each location in sequence is covered by one aircraft weight class. Constraints (6) and (7) allow the determination of the corresponding sequence for a given flow. Note that $c_{1}=0$, constraints (8) determine the time of location $l$. Constraints (8) force flow out of the source node, and constraints (9)-(11) assure that at each node $n_{i, l}$ the network flow is conserved. Each aircraft is traversed exactly once is guaranteed by constraints (12). The arc variables are linked to the assignment variables through constraints (13)-(16). The cumulative separation time in the aircraft wight class sequence at each location is determined by constraints (18) and (19). Constraints (19), (20) and (21) capture the realistic of landing time of aircraft at location $l$. The last constraints (22) are the nonnegativity and integrality constraints. Notice that not only the integrality constraints of second-stage variables $y_{i, l}^{s}$ but also of the first-stage variables $x_{k, l}$ can be relaxed, because the aircraft weight class and aircraft-to-location assignment can be captured in the flow variables.

\section{Solution Methodology}

Solving the problem directly (e.g., employing a state-andoff commercial solver such as CPLEX and Gurobi) is difficult for most practical problem, as large numbers of scenarios can yield extremely large-scale mixed-integer programs. Real-life situations involve a large number of scenarios which make it extremely difficult to solve SALP. To overcome this challenge, the SAA algorithm was applied. The main advantage of using the SAA algorithm is its ability to find near-optimal solutions while considering samples comprising a smaller number of scenarios. The SAA is a Monte Carlo simulationbased approach to solve stochastic programming problems (see Kleywegt et al. [18] for more details). The basic idea is simple indeed, i.e, a random sample is generated and the expected value function is approximated by the corresponding sample average function. It is used to compute lower and upper bounds of the expected recourse cost function. Therefore, the optimality gap and statistical confidence intervals on the quality of the approximate solutions can be evaluated simultaneously. In order to obtain a preestablished optimality gap, the procedure is repeated several times until the stopping criterion is satisfied.

The detailed steps involved in solving a SALP using SAA are described below.

\section{The SAA algorithm}

Step 1. Generate $M$ independent random samples each of size $N$ and solve the corresponding SAA problem:

$$
v_{N}=\min _{\mathbf{x} \in \mathbf{X}} c_{n}+\frac{1}{N} \sum_{n=1}^{N} Q\left(\mathbf{x}, \mathbf{T}^{n}\right)
$$

Let $v_{N}^{m}$ and $\hat{\mathbf{x}}_{N}^{m}$ be the corresponding optimal objective and optimal solutions, respectively; $m=1,2,3, \ldots, M$.

Step 2. Compute a lower bound and calculate the corresponding variance of this estimator.

$$
\begin{gathered}
\bar{v}_{N}^{M}=\frac{1}{M} \sum_{m=1}^{M} v_{N}^{m} \\
\sigma_{\bar{v}_{N}^{M}}^{2}=\frac{1}{M(M-1)} \sum_{m=1}^{M}\left(v_{n}^{m}-\bar{v}_{N}^{M}\right)^{2}
\end{gathered}
$$

The expected value of $\bar{v}_{N}^{M}$ is less than or equal to the optimal value $v^{*}$ of the original problem. Since $\bar{v}_{N}^{M}$ is an unbiased estimator of $\mathbb{E}\left[v_{N}\right]$ and $\mathbb{E}\left[\bar{v}_{N}^{M}\right] \leq v^{*}$. So we mean that $\bar{v}_{N}^{M}$ is a lower statistical bound for $v^{*}$ of the original problem and $\sigma_{\bar{v}_{N}^{M}}^{2}$ is an estimate of the variance of this estimator.

Step 3. Choose a feasible first-stage solution $\hat{\mathbf{x}} \in \mathbf{X}$ of the original problem, e.g., one of the solutions from $\hat{\mathbf{x}}_{N}^{m}$ and estimate the objective function value of the original problem using a different sample $N^{\prime}$. The true objective function value is given as: 


$$
v_{N^{\prime}}(\hat{\mathbf{x}})=c_{n}+\frac{1}{N^{\prime}} \sum_{n=1}^{N^{\prime}} Q\left(\hat{\mathbf{x}}, T^{n}\right)
$$

Especially, $N^{\prime}$ is chosen to be much larger than $N\left(N^{\prime} \gg\right.$ $N)$. The estimator $v_{N^{\prime}}(\hat{\mathbf{x}})$ is an unbiased estimator of $c_{n}+$ $\mathbb{E}\left[Q\left(\hat{\mathbf{x}}, T^{n}\right)\right]$. Since $\hat{\mathbf{x}}$ is a feasible solution to the true problem. The value $v_{N^{\prime}}(\hat{\mathbf{x}})$ is updated in each iteration if the obtained value is less than the value of the previous iteration. The variance of this estimate can be expressed as

$$
\sigma_{v_{N^{\prime}}(\hat{\mathbf{x}})}^{2}=\frac{1}{N^{\prime}\left(N^{\prime}-1\right)} \sum_{n=1}^{N^{\prime}}\left(c_{n}+Q\left(\hat{\mathbf{x}}, T^{n}\right)-v_{N^{\prime}}(\hat{\mathbf{x}})\right)^{2}
$$

Step 4. Compute an estimate of the optimality gap of the solution $\hat{\mathbf{z}}$ using the lower and upper bounds calculated in Steps 2 and 3, respectively, as follows:

$$
\operatorname{gap}_{N, M, N^{\prime}}(\hat{\mathbf{x}})=\frac{v_{N^{\prime}}(\hat{\mathbf{x}})-\bar{v}_{N}^{M}}{v_{N^{\prime}}(\hat{\mathbf{x}})}
$$

The estimated variance of the gap is given by

$$
\sigma_{\text {gap }}^{2}=\frac{\sigma_{\bar{v}_{N}^{M}}^{2}}{\left(v_{N^{\prime}}(\hat{\mathbf{x}})\right)^{2}}
$$

The confidence interval for the optimality gap can be calculated as

$$
v_{N^{\prime}}(\hat{\mathbf{x}})-\bar{v}_{N}^{M}+z_{\alpha}\left\{\sigma_{v_{N^{\prime}}(\hat{\mathbf{z}}, \hat{\eta})}^{2}+\sigma_{\bar{v}_{N}^{M}}^{2}\right\}^{0.5}
$$

with $z_{\alpha}=\Phi^{-1}(1-\alpha)$, where $\Phi(z)$ is the cumulative distribution of the standard normal distribution.

\section{NumericAl EXPERIMENTS}

In this section, we present computational experiments using a random-generation instance. The primary goal of these experiments is to study how stochastic arrival time influences the solutions. Another intention is to analyze the performance of the SAA algorithm. The SAA algorithm is fulfilled in Matlab 2014b and ILOG CPLEX 12.6 is utilized to solve the stochastic problem on a PC with Intel(R) Core(TM) i5$6500 \mathrm{CPU} 3.20 \mathrm{GHz}$ and $8 \mathrm{~GB}$ RAM. The input parameters used in this problem, the performance of the SAA method, and the analysis of computational results are presented in the following subsections.

\section{A. Scenario generation and data description}

Poisson arrival process (i.e., exponentially distributed interarrival times) is a realistic approximation of the inbound traffic of an airport. We choose an average inter-arrival time of 90 seconds. The separation times between leading and trailing aircrafts show in Table II. The weight class is generated according to it's probability distribution, we assure there are three aircraft weight classes (i.e., Heavy, Large and Small) and their probability distribution is identical. The resulting expected arrival times and aircraft classes for all 8 aircrafts are shown in Table III, where abbreviations $\mathrm{H}, \mathrm{L}$ and $\mathrm{S}$ represent the weight class Heavy, Large and Small respectively. Given the expected arrival times (i.e., mean values $\mu$ ), a realization sample arrival time for each aircraft can be generated according to it's probability distribution (e.g., normal distribution $N(\mu, \sigma)$ ), all of the realization arrival time for each aircraft consist of a particular scenario. We generate $|S|$ scenarios using the normal distribution $N(\mu, \sigma)$, where $\mu=$ ETA and $\sigma=\alpha \mu$, where $\alpha$ is a given parameter in the interval $[0,1]$. In this instance, we set $\alpha=0.2$.

TABLE III: Expected arrival times (EAT, in seconds) and weight classes (WC) of 10 aircrafts.

\begin{tabular}{lcccccccc}
\hline $\mathrm{N}$ & 1 & 2 & 3 & 4 & 5 & 6 & 7 & 8 \\
\hline EAT & 268 & 342 & 658 & 729 & 768 & 884 & 920 & 968 \\
$\mathrm{WC}$ & $\mathrm{L}$ & $\mathrm{H}$ & $\mathrm{S}$ & $\mathrm{L}$ & $\mathrm{H}$ & $\mathrm{H}$ & $\mathrm{S}$ & $\mathrm{S}$ \\
\hline
\end{tabular}

\section{B. Impact of stochastic arrival time on schedules}

TABLE IV: Computational results under different number of scenario

\begin{tabular}{cccc}
\hline Scenarios & Obj & Time(s) & Aircraft class sequence \\
\hline 5 & 1872 & 9.94 & L-S-H-S-S-L-H-H \\
6 & 1691 & 14.62 & L-S-S-L-S-H-H-H \\
7 & 1631 & 15.05 & L-S-S-L-H-H-H-S \\
8 & 1783 & 72.33 & L-S-H-H-L-S-S-H \\
9 & 1572 & 16.09 & L-S-H-L-S-S-H-H \\
10 & 1614 & 132.22 & L-S-H-L-S-S-H-H \\
12 & 1531 & 90.38 & L-S-H-L-S-S-H-H \\
15 & 1719 & 506.91 & L-S-H-L-S-S-H-H \\
\hline
\end{tabular}

Table IV shows the computational results of this instance solved by CPLEX directly. Obj indicates the total separation and delay times of 8 aircrafts. It can be observed from Table IV that as the number of scenario increases, the objective in general decreases and the running time increases in a nonlinear way. Moreover, it often makes better sense to assign a heavy class aircraft after the large and small. This can be explained by the fact that the separation time matrix is asymmetrical. For example, the separation time is 74 seconds between the small and the following heavy, however, 196 seconds of separation time have to be satisfied vice versa. The objective can reduce, only if the increased total delay time, which as a result of individual landing sequence shifting, can be offset by the decreased total separation time of the weight class order after exchanged.

\section{Numerical results of SAA algorithm}

In this subsection, the numerical experiments are conducted based on the above base case to illustrate the efficacy of the SAA algorithm and analyze the impact of sample size $N$ and replication $M$. The number of $N$ is progressively increased from 5 to 30 under $M=5$ and $M=10$, respectively. Table 
$\mathrm{V}$ details the results of numerical experiments, the first column shows the simple size (i.e., number of scenarios), columns two and three represent the statistical lower and upper bounds and their standard deviation (as percentage of the average value), last two columns indicate the optimality gap (in percentage) and the standard deviation of the gap.

It can be observed from Table $\mathrm{V}$ that as the sample size $N$ increases while $M$ is fixed, the optimality gap generally decreases and the standard deviation associated with lower, upper bounds and optimality generally drop as well. From Table $\mathrm{V}$, it can be found that as the replication $M$ increases while $N$ is fixed, the the statistical lower and upper bounds and their standard deviation generally decreases as well as a tighter optimality gap and a smaller corresponding standard deviation. On account of the lower and upper bounds obtained by the SAA algorithm are statistical in their nature, there is a chance that some lower bound values exceed those of the upper bounds. This results in a negative optimality gap (e.g., When $M=5, N=20$ ).

TABLE V: Statistical lower bound and upper bound obtained with $M=\{5,10\}$ and $N^{\prime}=500$.

\begin{tabular}{|c|c|c|c|c|c|c|}
\hline \multirow{2}{*}{$N$} & \multicolumn{2}{|c|}{ LB } & \multicolumn{2}{|c|}{ UB } & \multicolumn{2}{|c|}{ gap } \\
\hline & $\mathrm{E}$ & S.d(\%) & $\mathrm{E}$ & S.d(\%) & $\mathrm{E}(\%)$ & S.d \\
\hline \multicolumn{7}{|c|}{$(M=5)$} \\
\hline 5 & 1584.04 & 7.65 & 1782.88 & 7.78 & 11.15 & 40.17 \\
\hline 10 & 1804.24 & 14.59 & 1827.02 & 14.01 & 1.25 & 34.38 \\
\hline 20 & 1690.86 & 10.19 & 1644.97 & 9.28 & -2.79 & 60.32 \\
\hline 30 & 1492.43 & 7.64 & 1498.96 & 7.66 & 0.44 & 15.59 \\
\hline \multicolumn{7}{|c|}{$(M=10)$} \\
\hline 5 & 1812.10 & 6.72 & 1872.34 & 6.44 & 3.22 & 19.60 \\
\hline 10 & 1912.00 & 5.25 & 1949.17 & 5.05 & 1.91 & 21.45 \\
\hline 20 & 1645.76 & 7.07 & 1663.57 & 6.63 & 1.07 & 15.62 \\
\hline 30 & 1571.01 & 4.87 & 1585.24 & 4.70 & 0.88 & 11.04 \\
\hline
\end{tabular}

\section{CONCLUSION}

This article presents a two-stage stochastic mixed-integer programming model for the aircraft landing problem, which considers the uncertain of arrival time of aircraft. The objective of first-stage is to determine the best landing sequence of aircraft weight class (i.e., maximizing the throughput of runway). Given the solutions of first-stage, the individual aircraft will be assigned to the specific locations in the second-stage, which aims to minimize the total delay time of all aircrafts. In order to solve this stochastic problem, a SAA algorithm is developed. Statistical bounds on the optimal value and a near-optimal solution (with an optimality gap of less than $1 \%$ ) can be obtained for the SALP using the proposed SAA algorithm with 30 scenarios. In the future, there are several lines of work could be utilized to extend on this work. First, this stochastic formulation can be extend to multi-runways and mixed-mode runway. Second, the ground operations (e.g., taxing process), terminal air traffic control and gate assignment decision can be integrated. Third, the priority of flight and other objectives, such as total cost of operations and the fairness of schedules should be considered. Last, a more exact efficient algorithm can be developed to obtain solution in a reasonable computational time.

\section{ACKNOWLEDGMENT}

This work was supported by the National Natural Science Foundation of China (NSFC) under Grants 71428002, 71531011, 71571134, and 71771048. The work was also supported by the Fundamental Research Funds for the Central Universities.

\section{REFERENCES}

[1] J. A. Bennell, M. Mesgarpour, C. N. Potts, "Dynamic Scheduling of Aircraft Landings," European Journal of Operational Research, vol. 258, no. 1, pp. 315-327, 2017.

[2] A. Ghoniem, H. D. Sherali, H. Baik, "Enhanced Models for a Mixed Arrival-Departure Aircraft Sequencing Problem," INFORMS Journal on Computing, vol. 26, no. 3, pp. 514-530, 2014.

[3] J. Abela, D. Abramson, M. Krishnamoorthy, A. D. Silva, G. Mills, "Computing Optimal Schedules for Landing Aircraft," In Proceedings of the 12th national conference of the Australian Society for Operations Research, Adelaide, pp. 71-90, 1993.

[4] J. E. Beasley, M. Krishnamoorthy, Y. M. Sharaiha, D. Abramson, "Scheduling Aircraft Landings-the Static Case," Transportation Science, vol. 34, no. 2, pp. 180-197, 2000.

[5] H. Pinol, J. E. Beasley, "Scatter Search and Bionomic Algorithms for the Aircraft Landing Problem," European Journal of Operational Research, vol. 171, no. 2, pp. 439-462, 2006.

[6] D. Briskorn, R. Stolletz, "Aircraft Landing problems with Aircraft Classes," Journal of Scheduling, vol. 17, no. 1, pp. 31-45, 2014.

[7] R. G. Dear, Y. S. Sherif, "An Algorithm for Computer Assisted Sequencing and Scheduling of Terminal Area Operations," Transportation Research Part A, vol. 25, no. 2-3, pp. 129-139, 1991.

[8] H. Balakrishnan, B. G. Chandran, "Algorithms for Scheduling Runway Operations Under Constrained Position Shifting," Operations Research, vol. 58 , no. 6 , pp. $1650-1665,2010$.

[9] H. N. Psaraftis, "A Dynamic Programming Approach for Sequencing Groups of Identical Jobs," Operations Research, vol. 28, no. 6, pp. 13471359, 1980.

[10] A. Lieder, D. Briskorn, R. Stolletz, "A Dynamic Programming Approach for the Aircraft Landing Problem with Aircraft Classes," European Journal of Operational Research, vol. 243, no. 1, pp. 61-69, 2015.

[11] L. Bianco, P. Dell'Olmo, S. Giordani, "Minimizing Total Completion Time Subject to Release Dates and Sequence-dependent Processing Times," Annals of Operations Research, vol. 86, no. 1, pp. 393-415, 1999.

[12] J. E. Beasley, J. Sonander, P. Havelock, "Scheduling Aircraft Landing at London Heathrow Using a Population Heuristic," Journal of the Operational Research Society, vol. 52, no. 5, pp. 483-493, 2001.

[13] A. Faye, "Solving the Aircraft Landing Problem with Time Discretization Apporach," European Journal of Operational Research, vol. 242, no. 3, pp. 1028-1038, 2015.

[14] L. H. Lee, C. U. Lee, Y. P. Tan, "A Multi-objective Genetic Algorithm for Robust Flight Scheduling Using Simulation," European Journal of Operational Research, vol. 177, no. 3, pp. 1948-1968, 2007.

[15] K. K. H. Ng, C. K. M. Lee, F. T. S. Chan, Y. Qin, "Robust Aircraft Sequencing and Scheduling Problem with Arrival/Departure Delay Using the Min-max Regret Approach," Transportation Research Part E, vol. 106, no. 2, pp. 115-136, 2017

[16] J.C. Picard, M. Queyranne, "The Time-dependant Traveling Salesman Problem and It's Application to the Tardiness Problem in One-machine Scheduling," Operations Research, vol. 26, no. 1, pp. 86-110, 1978.

[17] L. P. Bigras, M. Gamache, G. Savard, "The Time-Dependent Traveling Salesman Problem and Single Machine Scheduling Problems with Sequence Dependent Setup Times," Discrete Optimization, vol. 5, no. 4, pp. 685-699, 2008

[18] A. J. Kleywegt, A. Shapiro, T. Homem-De-Mello, "The sample average approximation method for stochastic discrete optimization," SIAM Journal on Optimization, vol. 12, no.2, pp. 479-502, 2002. 